\title{
Evaluation of rare earth doped silica sub-micrometric spheres as optically controlled temperature sensors
}

\author{
P. Haro-González, ${ }^{1}$ L. Martínez Maestro, ${ }^{2}$ M. Trevisani, ${ }^{1}$ S. Polizzi, ${ }^{3}$ D. Jaque,${ }^{2}$ \\ J. García Sole, ${ }^{2}$ and M. Bettinelli ${ }^{1}$ \\ ${ }^{1}$ Laboratorio di Chimica dello Stato Solido, DB, Università di Verona and INSTM, UdR Verona, \\ Ca' Vignal, Strada Le Grazie 15, I-37134 Verona, Italy \\ ${ }^{2}$ Fluorescence Imaging Group, Departamento de Física de Materiales, C-IV, Universidad Autónoma de \\ Madrid, C/Francisco Tomás y Valiente 7, E-28049 Madrid, Spain \\ ${ }^{3}$ Dipartimento di Scienze Molecolari e Nanosistemi, Università Ca' Foscari Venezia, Dorsoduro 2137, \\ I-30123 Venezia, Italy
}

(Received 9 June 2012; accepted 9 August 2012; published online 12 September 2012)

\begin{abstract}
We report on the evaluation of rare earth $\left(\mathrm{Er}^{3+}, \mathrm{Eu}^{3+}\right.$, and $\mathrm{Tb}^{3+}$ ions) $\mathrm{SiO}_{2}$ sub-micrometric spheres as potential optically controllable temperature sensors. Details about fabrication, optical manipulation and spectroscopic characterization of the sub-micrometric spheres are presented. The fluorescence properties of the micros-spheres in the biological range $\left(25-60^{\circ} \mathrm{C}\right)$ have been systematically investigated. From this systematic study, the thermal resolution potentially achieved in each case has been determined and compared to previous works. (C) 2012 American Institute of Physics. [http://dx.doi.org/10.1063/1.4751349]
\end{abstract}

\section{INTRODUCTION}

Thermal sensing at the micro/nano scale is one of the most challenging topics that the scientific community is nowadays facing. ${ }^{1-4}$ It is required in many fields, ranging from integrated photonics (where the unavoidable light-induced thermal loading could lead to a drastic deterioration in the performance of active optical circuits) to micro/nano electronics (where the appearance of Joule-induced "hot-spots" is one of the most common causes of fatal failure). ${ }^{5,6}$ This can be extended to the case of the new generation opto-fluidic devices which nowadays constitute lab-on-a-chip platforms with numerous applications in life sciences, such as cell manipulation and sorting. ${ }^{7,8}$ In these applications, light is used to manipulate living cells that propagate within micro-fluidics through the use of optical forces. ${ }^{9}$ In this case, residual absorption of fluids at laser radiation wavelengths can cause non negligible thermal loadings that could lead to malfunctioning of the device due to the appearance of convection currents or to alterations in the cell dynamics. ${ }^{10}$ Therefore, an accurate knowledge of the local temperatures achieved within the micro-channels becomes essential for a complete understanding of the device performance as well as for the determination of the working limits of the device. In addition to opto-fluidic devices, high resolution and high sensitivity of thermometry are also being required in biomedicine. It is well known that in any bio-system, temperature plays a crucial role in determining its dynamics and properties. ${ }^{1,2}$ For example, temperature is known to be one of the critical parameters affecting cell division rates and hence, determining the rate of tissue growth. ${ }^{11}$ In these last potential applications, thermal sensing must be achieved with the minimum perturbation of the living cell, in such a way that attaining the thermal information does not alter the normal development of the cell activity.

Motivated by the numerous fundamental and practical applications, many research groups have presently focused their efforts on the development of diverse techniques capable of achieving thermal sensing with sub-micrometric resolution in both micro-fluidics and bio systems. ${ }^{12,13}$ Most of these techniques are based on the incorporation into the system to be measured (microfluidics or living cells) of submicrometric sized materials, whose structural or optical properties are strongly temperature dependent in such a way that they constitute sub-micrometric thermal sensors. ${ }^{4,12-15}$ The list of sub-micrometric materials already used for thermal sensing at the nanoscale has been boosted by the fast development of Nanotechnology and nowadays includes a large variety of materials such as carbon nanotubes, ${ }^{16}$ silver nanospheres, ${ }^{17}$ organic dyes, ${ }^{18}$ and quantum dots. ${ }^{19,20}$ However, such approaches also exhibit some drawbacks, since the nanomaterials pervade the whole measurement volume. For applications including microreactor (where the presence of organic dye could interfere with chemical reactions) and biological applications involving the use of permeable cells (where toxicity plays a crucial role), the massive and non-selective incorporation of nano-materials could be problematic.

To overcome this drawback, the use of optical tweezers has been revealed as a powerful tool, because it allows the use of a single particle whose position over the system to be thermally imaged can be controlled by optical forces. Indeed, optical tweezers have been used previously to manipulate objects within micro-fluidic devices, for example, to analyze flow fields by measuring the displacement of trapped beads ${ }^{21}$ as micro-pumps or microvalves,${ }^{22}$ for cell manipulation alone ${ }^{23}$ or associated with a visualization technique such as fluorescence microscopy, ${ }^{24}$ super resolution microscopy ${ }^{25}$ or Raman spectroscopy. ${ }^{26}$ In addition, the light-controlled motion and positioning of sub-micrometric size objects has been widely used in single-cell studies allowing, for example, Raman studies of single cell or dynamical studies of the mechanical properties of cells. 
Optical Tweezers Nanothermometry (OTNth) consists on the simultaneous optical trapping and excitation of submicrometric luminescent spheres showing a strongly temperature dependent luminescence. The continuous scanning of the trapping laser over the system to be imaged, in combination with the analysis of the luminescence sub-micrometric spheres, provides the desired thermal image. Due to the relative low laser powers required for optical trapping of submicrometric spheres, thermal sensing achieved by OTNth can be obtained with a minimum perturbation of the medium. Of course, OTNth implies the use of sub-micrometric spheres with an outstanding solubility in water and, simultaneously, showing a luminescence that is strongly influenced by small changes in the local environment. In this sense, a possible approach is to use rare earth $\left(\mathrm{RE}^{3+}\right)$ doped silica sub-micrometric spheres. Rare earth ions have been already demonstrated to be valuable luminescence-based optical sensors in a great variety of systems, including crystals, glasses and complexes. ${ }^{13,27,28}$ Despite its potential interest, the evaluation of rare earth doped silica microspheres as suitable laser controlled optical sensors has been very limited so far.

In this work, we have evaluated the potential use of $\mathrm{RE}^{3+}$ doped $\mathrm{SiO}_{2}$ sub-micrometric spheres as an optically controlled thermal sensor. In a first step, we synthesized submicrometric spheres with a reasonable size dispersion doped with different $\mathrm{RE}^{3+}$ ions $\left(\mathrm{Er}^{3+}, \mathrm{Eu}^{3+}\right.$, and $\mathrm{Tb}^{3+}$ ions) which can be dispersed in aqueous solution. Then, we have verified that the dispersion of the sub-micrometric spheres is good enough to ensure their manipulation and control in liquids by using optical tweezers. Finally, the temperature dependence of the sub-micrometric sphere luminescence has been systematically studied in the biological range $\left(25-60^{\circ} \mathrm{C}\right)$. From this analysis, we have determined the thermal resolution achieved for different rare earth ions as well as the most suitable fluorescence parameter that should be used for thermal sensing.

\section{EXPERIMENTAL}

Sub-micrometer spheres of silica doped with $0.5 \mathrm{~mol}$. \% of efficient $\mathrm{RE}^{3+}$ ions $\left(\mathrm{Eu}^{3+}, \mathrm{Tb}^{3+}\right.$, and $\mathrm{Er}^{3+}$ ) were synthesized by base-catalyzed hydrolysis of tetraethoxysilane (TEOS) via the well-known Stöber process ${ }^{29}$ i.e., hydrolysis of TEOS in an ethanol solution containing water and ammonia.
In a typical experiment $0.17 \mathrm{~mol} / \mathrm{l}$ of TEOS (Aldrich, 98 wt. $\%), 7.7 \mathrm{~mol} / \mathrm{l}$ of distilled water $(18.2 \mathrm{M} \Omega$ ), and $1.7 \mathrm{~mol} / \mathrm{l}$ of $\mathrm{NH}_{3}$ (Aldrich, $30 \mathrm{wt}$. \%) were used to produce monodisperse silica spheres. The doping of silica spheres with the $\mathrm{RE}^{3+}$ ions was obtained by adding the $\mathrm{RE}\left(\mathrm{NO}_{3}\right)_{3} \cdot 5 \mathrm{H}_{2} \mathrm{O}$ (Aldrich, 99.999\%). The solution was stirred overnight at room temperature, obtaining final milky colloids which were precipitated by centrifugation $(5000 \mathrm{rpm}, 10 \mathrm{~min})$ and rinsed three times in ethanol in an ultrasonic bath. The spheres were then dried and annealed in a furnace at $900^{\circ} \mathrm{C}$ for $2 \mathrm{~h}$. Submicrometer spheres were dispersed in distilled water $(1 / 1000)$.

Transmission electron microscopy (TEM) images were obtained with a JEOL 3010 high resolution electron microscope $(0.17 \mathrm{~nm}$ point-to-point), operating at $300 \mathrm{kV}$, equipped with a Gatan slow-scan CCD camera (model 794) and an Oxford Instrument EDS microanalysis detector (Model 6636).

Luminescence measurements were carried out upon $\mathrm{cw}$ excitation with $405 \mathrm{~nm}$ and $488 \mathrm{~nm}$ diode lasers. The beam was focused into the colloidal dispersion by using a $50 \times$ microscope objective with a numerical aperture of 0.55 . The samples were positioned onto a heater plate in order to obtain their luminescence response as a function of the temperature. The emitted luminescence was analyzed with a $0.5 \mathrm{~m}$ monochromator (SPEX 0.5 M) connected to an imaging CCD camera.

Demonstration of optical trapping of the $\mathrm{RE}^{3+}$ submicrometric spheres was achieved in a homemade optical trapping set-up. The colloidal dispersion was placed within micro-channel provided by Ibidi $\mathrm{GmbH}$. The microchannel was $120 \mu \mathrm{m}$ depth and $6 \mathrm{~mm}$ width. A $980 \mathrm{~nm}$ fiber coupled diode was focused into the micro-chamber using a $100 \times$, $0.8 \mathrm{NA}$ objective. The size of the $980 \mathrm{~nm}$ trapping spot was estimated to be close to $1 \mu \mathrm{m}$. The position and controlled motion of the dispersed micro-spheres was monitored by a CCD camera.

\section{RESULTS AND DISCUSSION}

The typical TEM image of the sub-micrometric spheres used in this work is given in Figure 1(a). It reveals that the silica spheres exhibit regular, smooth shape, and uniform size. The particles are all non-aggregated with narrow size
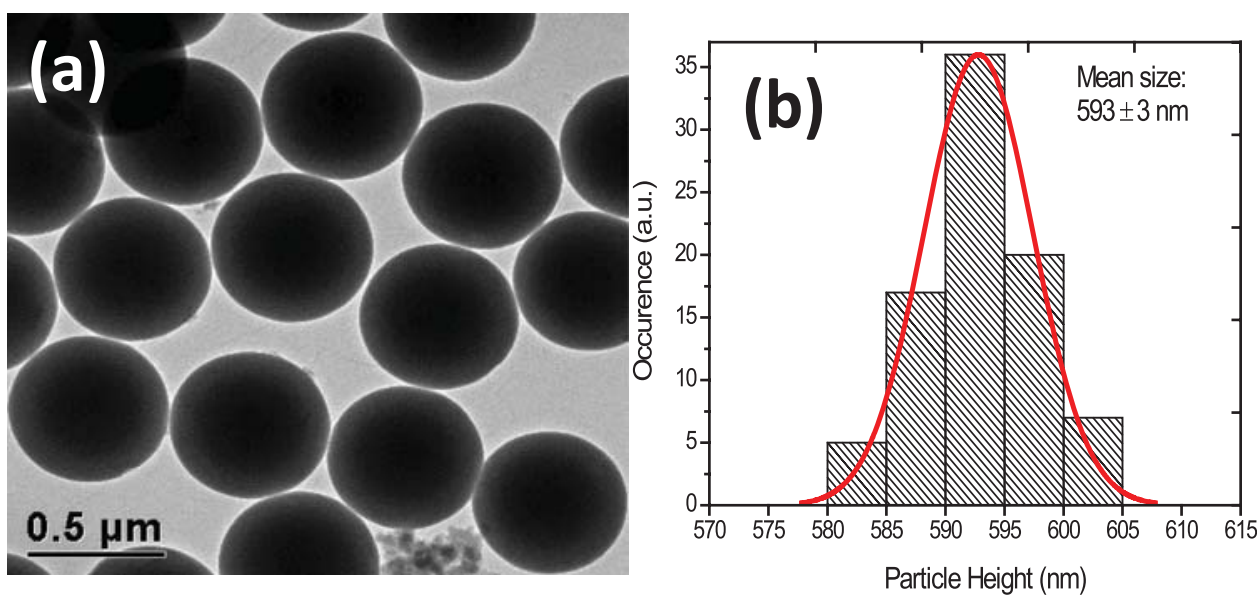

FIG. 1. (a) TEM micrograph of $\mathrm{RE}^{3+}$ doped sub-micrometer spheres after annealing. (b) Histogram of the height distribution of the microspheres; the red line is the Gaussian fit of the histogram and results in a mean size of $593 \pm 3 \mathrm{~nm}$ of the particles. 


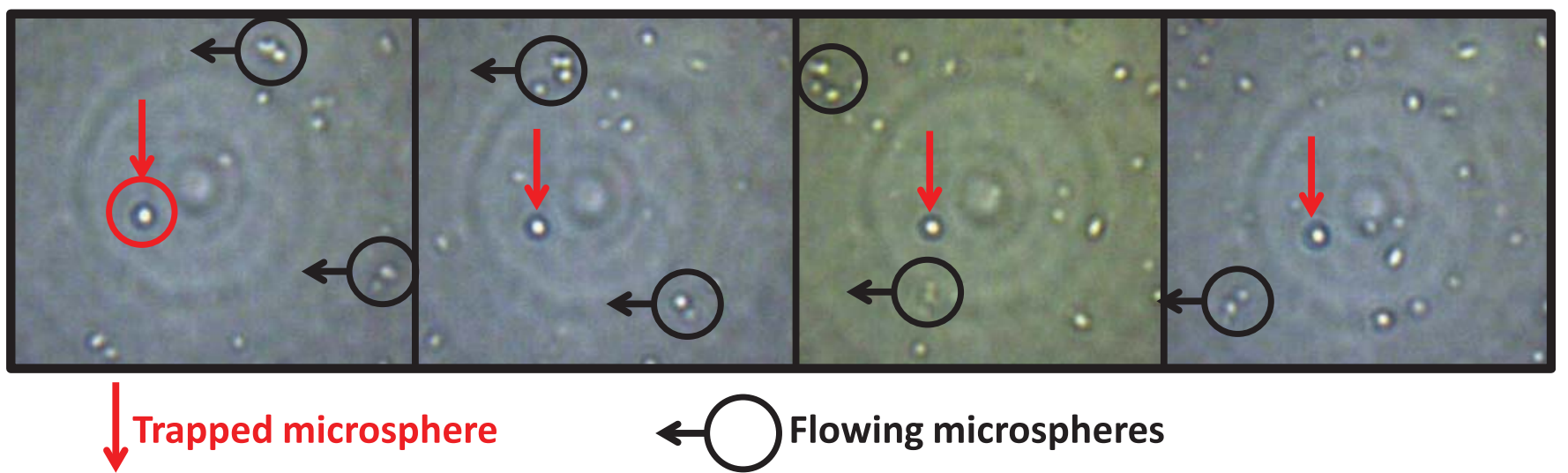

FIG. 2. Evidence of trapping of the $\mathrm{RE}^{3+}$ doped microspheres. The sequence represents the movement of a single sphere (marked in a red-circle in the first image and only with a red-arrow in the rest) in a horizontal line while the rest of the spheres (marked with black-circles) continues with their free movement, with a trapping threshold of $10 \mathrm{~mW}$.

distribution (Figure 1(b)) characterized by a mean diameter of $593 \mathrm{~nm}$ and a size poly-dispersion of $\sigma \approx 2 \%$.

Figure 2 evidences the optical trapping and manipulation of our sub-micrometric spheres when dispersed in an aqueous solution. In this figure, a series of images from the tweezers-control camera illustrating the capture (and positioning) of a single sphere in the micro-fluidic-channel is shown. The pictures show an optically trapped sphere (marked with an arrow) in the presence of an externally induced horizontal flux. It is clear how the trapped sphere remains at the same position (position of the laser spot) whereas the non-trapped spheres (some of them market with a circle) move horizontally (right to left sense) following the externally established flux. The spheres can be trapped at powers just above the lasing threshold $(\approx 10 \mathrm{~mW})$. As this trapping laser could perturb device operation or produce
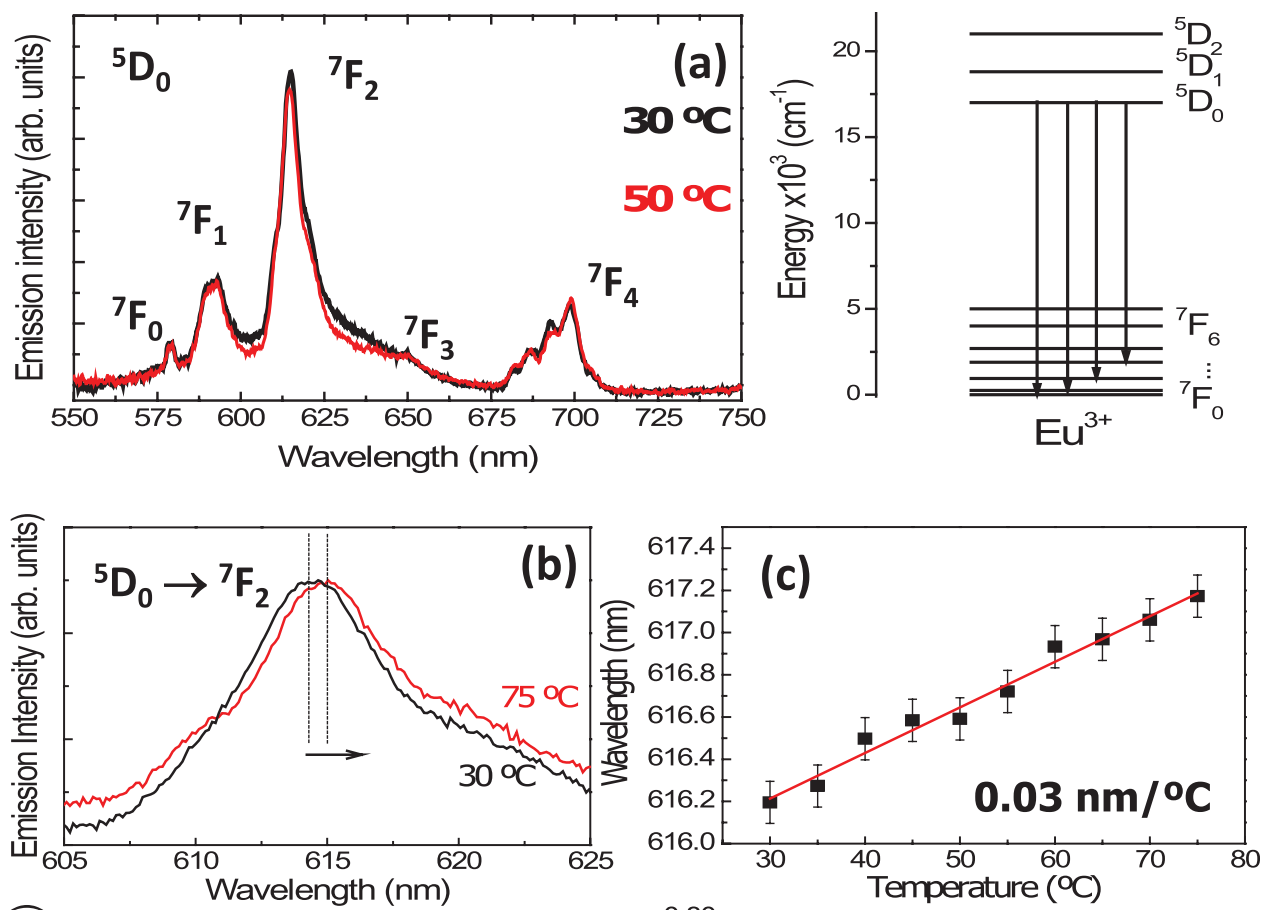

FIG. 3. (a) Emission spectra of the $\mathrm{Eu}^{3+}$ doped $\mathrm{SiO}_{2}$ microspheres after optical excitation at $405 \mathrm{~nm}$. (b) Detail of the $\mathrm{Eu}^{3+}$ transition centred at around $615 \mathrm{~nm}$ at low and high temperature, (c) shift of this peak with temperature. (d) Detail of the $\mathrm{Eu}^{3+}$ transition centred at around $700 \mathrm{~nm}$ at $30^{\circ} \mathrm{C}$ and $75^{\circ} \mathrm{C}$, (e) intensity ratio of the two indicated peaks with temperature.
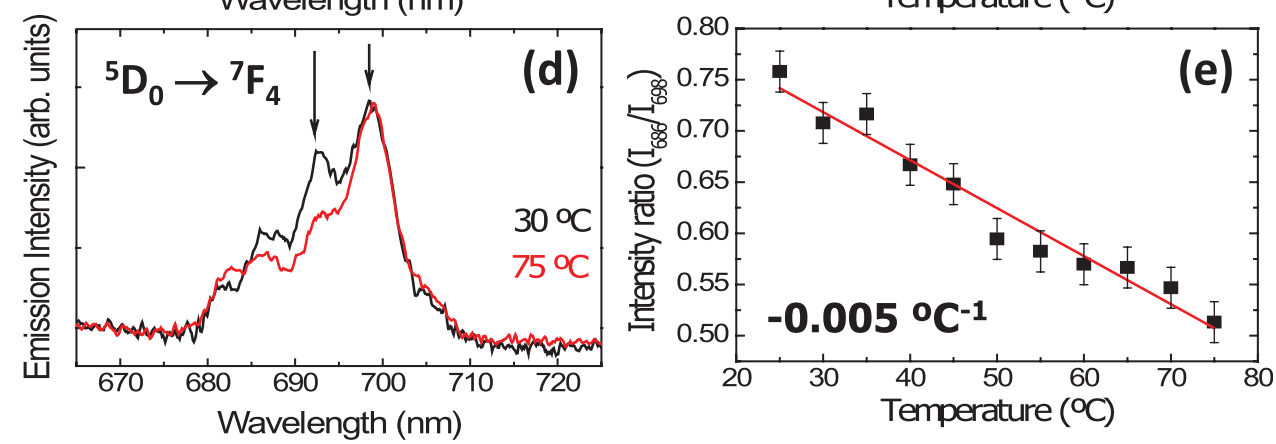

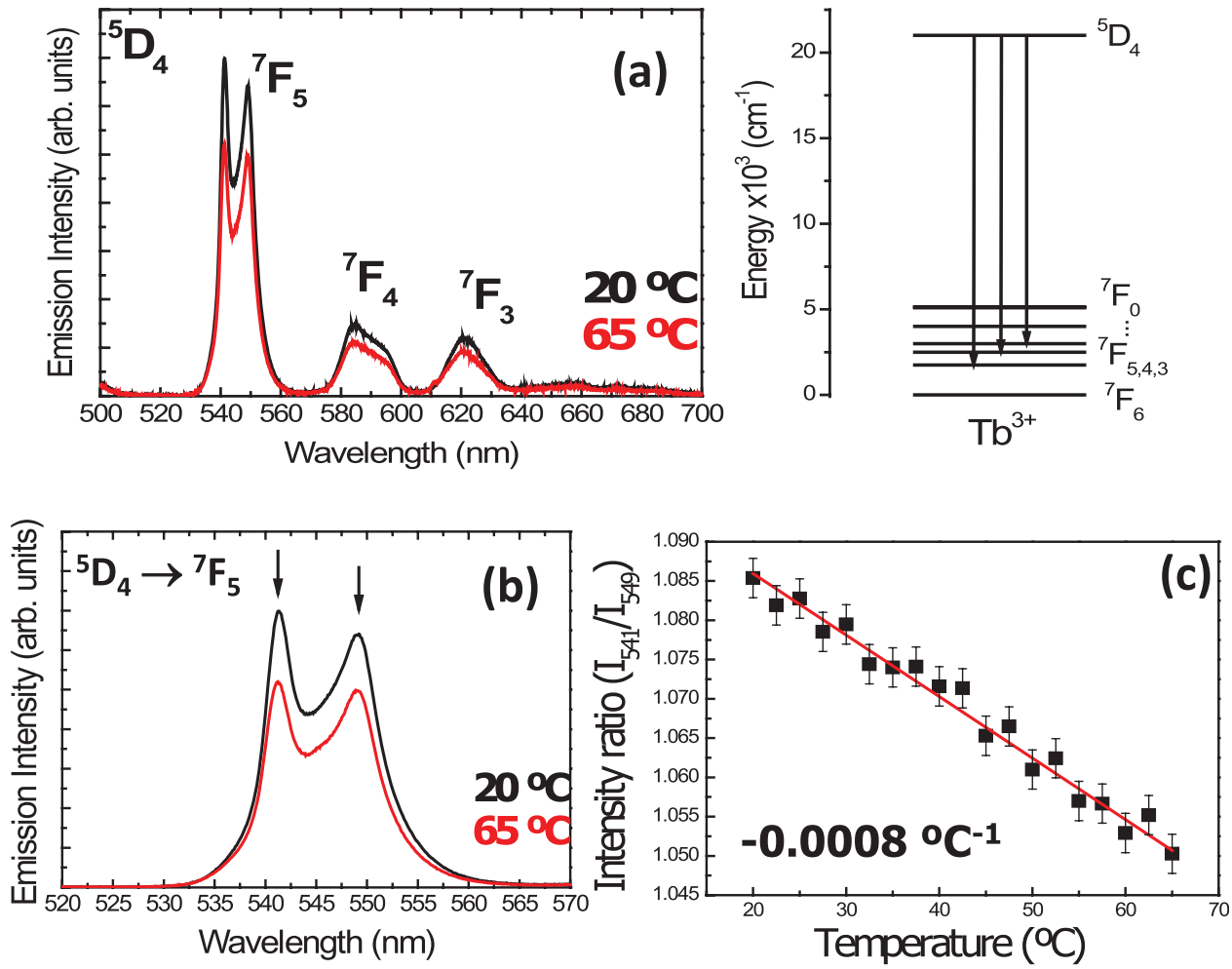

FIG. 4. (a) Emission spectra of the $\mathrm{Tb}^{3+}$ doped $\mathrm{SiO}_{2}$ microspheres after optical excitation at $488 \mathrm{~nm}$. (b) Detail of the $\mathrm{Tb}^{3+}$ transition centred at around $545 \mathrm{~nm}$ at $20^{\circ} \mathrm{C}$ and $65^{\circ} \mathrm{C}$, (c) ratio of the two indicated peaks with temperature. damages in biological samples, ${ }^{18}$ the laser induced heating at the trap has been estimated for this power according to a model reported by Mao et al. ${ }^{30}$ In this model, the spatial variation of temperature of a microfluidic locally heated by tightly focused laser beam is estimated. The temperature increment at focus is given by

$$
\Delta T_{\text {focus }}=\frac{P_{\text {in }} \alpha_{w}}{2 \pi K} \operatorname{Ln} \frac{D}{w_{l}},
$$

where $P_{i n}$ is the laser incident power, $D$ is the height of the $\mu$-chamber (120 $\mu \mathrm{m}$ in our case), $\alpha_{w}$ the absorption coefficient of water at laser wavelength $\left(0.5 \mathrm{~cm}^{-1}\right.$ in our case $), K$ the thermal conductivity of water, and $w_{l}$ the laser beam radius (estimated to be $0.7 \mu \mathrm{m}$ in our case). Thus, the heating due to the tweezers beam was estimated to be $\Delta \mathrm{T}_{\text {focus }}=0.006^{\circ} \mathrm{C}$. Indeed, this small temperature increase should not perturb the device operation, would not damage biological samples and does not affect the temperature measurement.

Once it has been confirmed that our sub-micrometric spheres can be trapped and hence optically manipulated with a low (non perturbative) laser power, we can evaluate them as optical sensors. For this purpose, we have performed a systematic study of their luminescence properties in the temperature range $30-75^{\circ} \mathrm{C}$, i.e. around the biological range. Optical thermometry is based on the analysis of temperature-induced changes in the optical properties of materials and includes a great variety of techniques. In the case of our spheres, the relationship between temperature and luminescence properties has been systematically investigated in order to achieve thermal sensing from spatial and spectral analysis. The sensitivity " $\mathrm{S}$ " of a thermometric system is defined as the variation of the measured parameter (MP) with temperature ${ }^{31}$

$$
S=\frac{1}{M P}\left(\frac{d M P}{d T}\right) .
$$

In Figures 3(a), 4(a), and 5(a) the emission spectra of the $\mathrm{Eu}^{3+}, \mathrm{Tb}^{3+}$, and $\mathrm{Er}^{3+}$ doped sub-micrometric spheres at two temperatures are presented. The corresponding energy level diagrams (showing only the energy levels of interest) have been also included. It is evident from these figures that, in all cases, the luminescence spectra are affected by temperature variation within the biological range. In all cases, the emission intensity of the $\mathrm{RE}^{3+}$ ions decreases with increasing the temperature, indicating the presence of a phonon assisted thermal quenching process. This general temperature induced luminescence reduction can, in principle, be used as temperature indicator. However the luminescence intensity as a thermal indicator has several drawbacks as it could depend on external factors, such as fluctuations in the laser excitation intensity. Therefore, the evaluation of the $\mathrm{RE}^{3+}$ doped microspheres as a thermometer is better based on the analysis of temperature changes in parameters such as spectral position and bandshape.

Figure 3(a) shows the emission spectra of $\mathrm{Eu}^{3+}$ doped sub-micrometric spheres/water solution at $30{ }^{\circ} \mathrm{C}$ and $50^{\circ} \mathrm{C}$ after optical excitation at $405 \mathrm{~nm}$. These emission bands were assigned to the ${ }^{5} \mathrm{D}_{0} \rightarrow{ }^{7} \mathrm{~F}_{\mathrm{J}}$ (with $\mathrm{J}$ from 0 to 4 ) transitions. Mainly, the red emission centered at around $615 \mathrm{~nm}$ is expected to be temperature dependent due to its large sensitivity to environmental effects in the vicinity of the $\mathrm{Eu}^{3+}$ ions. $^{32,33}$ This fact is shown in Figure 3(b), where the luminescence spectra for the $\mathrm{Eu}^{3+}:{ }^{5} \mathrm{D}_{0} \rightarrow{ }^{7} \mathrm{~F}_{2}$ transition at $30^{\circ} \mathrm{C}$ and $75^{\circ} \mathrm{C}$ is given. A red-shift of the peak can be appreciated as the temperature increases. Figure 3(c) shows that this shift varies linearly with temperature with a slope of $0.030 \pm 0.002 \mathrm{~nm}^{\circ} \mathrm{C}^{-1}$. Another interesting point to note is 

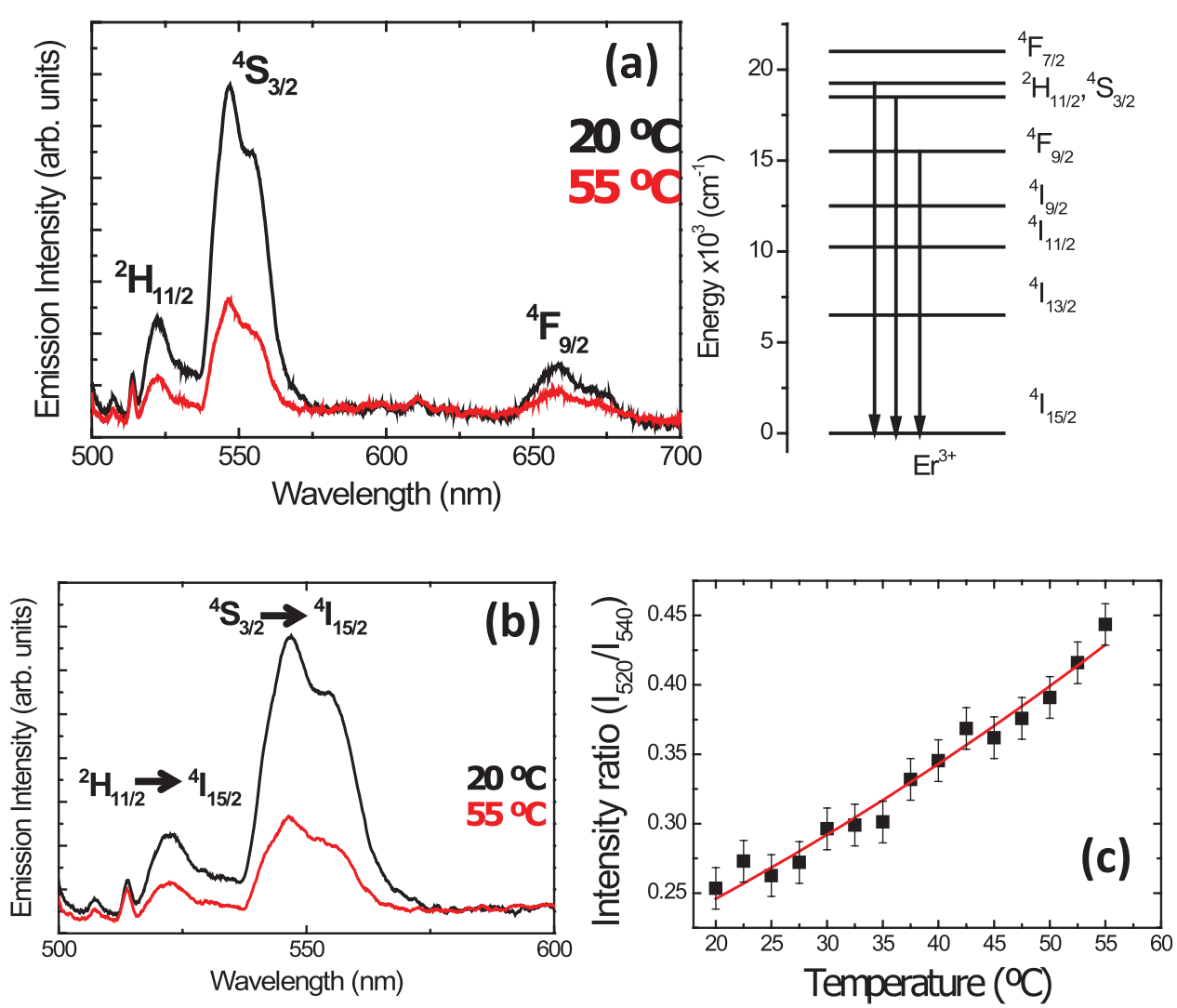

FIG. 5. (a) Emission spectra of the $\mathrm{Er}^{3+}$ doped $\mathrm{SiO}_{2}$ microspheres after optical excitation at $488 \mathrm{~nm}$. (b) Detail of the thermalized levels of $\mathrm{Er}^{3+}$ ions at $20^{\circ} \mathrm{C}$ and $55^{\circ} \mathrm{C}$, (c) intensity ratio of the two emission band. the thermal sensitivity of the Eu ${ }^{3+}:{ }^{5} \mathrm{D}_{0} \rightarrow{ }^{7} \mathrm{~F}_{4}$ transition band presented in Figure 3(d). This transition has been monitored with the temperature and it is noted that the intensity ratio of the two main peaks of this transition (indicated with arrows in Figure 3(d)) may be used to estimate the temperature over a wide range with appreciable sensitivity. Figure 3(e) shows that the intensity ratio of these peaks $\left(\frac{I_{686}}{I_{69}}\right)$ varies linearly with temperature at a rate of $0.0050 \pm 0.0003^{\circ} \mathrm{C}^{-1}$.

Therefore, data included in Figs. 3(b) and 3(d) indicate that thermal sensing can be achieved by using europium doped micro-spheres by means of ratiometric or spectral analysis of its luminescence spectrum. In Table I, a summary of several $\mathrm{Eu}^{3+}$ doped materials, with their corresponding sensitivities (expression (2)), together with our results is presented. First, by comparing the spectral shift and band-shape results obtained for our spheres, the superior sensitivity of the spectral shift technique, which is due to the hypersensitive $\mathrm{Eu}^{3+}$ : ${ }^{5} \mathrm{D}_{0} \rightarrow{ }^{7} \mathrm{~F}_{2}$ transition is clear. To the best of our knowledge, no work reporting on the thermal sensing capabilities of $\mathrm{Eu}^{3+}$ doped sub-micrometric spheres has been published up to now. By comparing the different sensitivities reported, we conclude that our $\mathrm{Eu}^{3+}$ doped sub-micrometric spheres can be proposed as a good candidate for thermal sensing in microfluidic, due to their good sensitivity together with the additional advantage that they can be easily trapped without perturbation of the medium.

Figure 4(a) shows the emission spectra of $\mathrm{Tb}^{3+}$ doped microspheres at $20^{\circ} \mathrm{C}$ and $65^{\circ} \mathrm{C}$ after optical excitation at $488 \mathrm{~nm}$. All the emission bands correspond to the visible luminescence from the ${ }^{5} \mathrm{D}_{4}$ state to the ${ }^{5} \mathrm{~F}_{\mathrm{J}}$ (with $\mathrm{J}$ from 3 to 5 ) states of the $\mathrm{Tb}^{3+}$ ion. As can be seen in this Figure, there is a decrease of $\mathrm{Tb}^{3+}$ luminescence intensity with increasing temperature indicating again the thermal sensitivity of these ions. This behaviour is probably due to weak multiphonon relaxation. The $\mathrm{Tb}^{3+}:{ }^{5} \mathrm{D}_{4} \rightarrow{ }^{7} \mathrm{~F}_{5}$ transition, presented in Figure 4(b) is particularly sensitive. The intensity ratio of the two peaks $(541 \mathrm{~nm} 548 \mathrm{~nm})$ changes linearly with the temperature at a rate of $0.00080 \pm 0.00004{ }^{\circ} \mathrm{C}^{-1}$ (see Figure 4(c)). According to Eq. (2), at the physiological temperature of $37^{\circ} \mathrm{C}$, the sensitivity of $\mathrm{Tb}^{3+}$ doped $\mathrm{SiO}_{2}$ microspheres reaches a value of $0.0007^{\circ} \mathrm{C}^{-1}$. Unfortunately, the sensitivities reported in several $\mathrm{Tb}^{3+}$ doped materials by other researchers ${ }^{34,35}$ (around

TABLE I. Different sensitivities and their corresponding sensing techniques for several $\mathrm{Eu}^{3+}$ doped materials.

\begin{tabular}{|c|c|c|c|c|}
\hline $\mathrm{Eu}^{3+}$ & Technique & $\mathrm{S}\left(37^{\circ} \mathrm{C}\right) \times 10^{-3}{ }^{\circ} \mathrm{C}^{-1}$ & $\mathrm{~T}$ range & Ref \\
\hline DT solution & Decaytime & 22 & $10-45^{\circ} \mathrm{C}$ & 45 \\
\hline Tellurite glass & FIR & 23 & $27-277^{\circ} \mathrm{C}$ & 46 \\
\hline $\mathrm{BaB}_{4} \mathrm{O}_{7}$ nanocrystals & FIR & 24 & $-250-400^{\circ} \mathrm{C}$ & 47 \\
\hline lanthanum oxysulphide & Decaytime & 20 & $5-60{ }^{\circ} \mathrm{C}$ & 48 \\
\hline solution & intensity & 30 & $20-50^{\circ} \mathrm{C}$ & 1 \\
\hline \multirow[t]{2}{*}{$\mathrm{SiO}_{2}$ microspheres } & Spectral shift & 30 & $30-75^{\circ} \mathrm{C}$ & This work \\
\hline & Band-shape & 7 & $30-75^{\circ} \mathrm{C}$ & This work \\
\hline
\end{tabular}


$0.01{ }^{\circ} \mathrm{C}^{-1}$ ) show much better values comparing with our result.

One of the most common used trivalent rare earth ion for thermal sensing is $\mathrm{Er}^{3+}$ (see emission in Figure 5(a)). ${ }^{36-44}$ In this case, the local temperature is extracted from the analysis of the relative intensities of the visible emission bands at around 520 and $550 \mathrm{~nm}$. These bands are generated from thermally coupled states of the $\mathrm{Er}^{3+}$ ions; ${ }^{4} \mathrm{~S}_{3 / 2}$ and ${ }^{2} \mathrm{H}_{11 / 2}$ (see Figure 5(b)). The temperature dependent ratio of these band intensities is temperature dependent on excitation since the emitted intensities are proportional to the population of the corresponding excited states. This relative population between the two levels, $\mathrm{R}$, follows a Boltzmann-type population distribution: ${ }^{31}$

$$
R=\frac{I_{31}}{I_{21}}=\frac{\omega_{31}^{R} g_{3} h \nu_{3}}{\omega_{21}^{R} g_{2} h \nu_{2}} \exp \left(-\frac{E_{32}}{K T}\right)=C \exp \left(-\frac{E_{32}}{K T}\right),
$$

where $\mathrm{K}$ is the Boltzmann constant, $\mathrm{E}_{32}$ the energy gap between the ${ }^{2} \mathrm{H}_{11 / 2}$ and ${ }^{4} \mathrm{~S}_{3 / 2}$ excited states levels, $g_{3}, g_{2}$ are the degeneracies $(2 J+1)$ of the levels, $\omega^{\mathrm{R}}{ }_{31}$ and $\omega^{\mathrm{R}}{ }_{21}$ are, respectively, the spontaneous emission rates from the $\mathrm{E}_{3}$ $\left({ }^{2} \mathrm{H}_{11 / 2}\right)$ and $\mathrm{E}_{2}\left({ }^{4} \mathrm{~S}_{3 / 2}\right)$ states to the $\mathrm{E}_{1}\left({ }^{4} \mathrm{I}_{15 / 2}\right)$ ground state.

Small changes in the local temperature cause relevant changes in the Boltzmann population of the $\mathrm{Er}^{3+}$ green emitting levels and this leads to measurable changes in the intensity of the $520 \mathrm{~nm}$ and $540 \mathrm{~nm}$ emission bands (see Figure 5(c)). According to our results, at the physiological temperature of $37^{\circ} \mathrm{C}$, the sensitivity of $\mathrm{Er}^{3+}$ doped $\mathrm{SiO}_{2}$ submicrometric spheres reaches a value of $0.016{ }^{\circ} \mathrm{C}^{-1}$. In Table II, we present a summary of results for the $\mathrm{Er}^{3+}$ doped spheres reported in the literature, together with other silica bulk samples. The emission intensity ratio (FIR) parameters and the sensitivity at $37^{\circ} \mathrm{C}$ are compared for these different systems. From Eq. (3), it is clear that the materials with larger $\mathrm{E}_{32}$ energy separations between emitting states show larger sensitivities. This is the case of our $\mathrm{SiO}_{2}$ sub-micrometric spheres in which the highest sensitivity value is found.

To summarize all the results, Table III shows the thermal sensitivities as well as the used techniques for the three investigated systems. The emission spectra of all the $\mathrm{RE}^{3+}$ doped sub-micrometric spheres here investigated are affected by temperature variations within the biological range. They have been evaluated as temperature sensors based on the most important changes produced in their fluorescent features. The sensitivities obtained in each case have been compared. It is clear from these results, that the $\mathrm{Eu}^{3+}$ spheres display the high-

TABLE II. Emission intensity ratio parameters and thermal sensitivities $\left(37^{\circ} \mathrm{C}\right)$ for different $\mathrm{Er}^{3+}$ doped material (spheres and $\mathrm{SiO}_{2}$ glass samples).

\begin{tabular}{lccccc}
\hline \hline Material & $\begin{array}{c}\text { Radius sphere } \\
(\mu \mathrm{m})\end{array}$ & $\mathrm{C}$ & $\Delta \mathrm{E} / \mathrm{K}$ & $\left(37^{\circ} \mathrm{C}\right) \times 10^{-3}{ }^{\circ} \mathrm{C}^{-1}$ & Ref. \\
\hline $\mathrm{SiO}_{2}$ & 0.6 & 45.2 & 1528 & 16 & This work \\
$\mathrm{Phosphate}$ & 17 & 14.4 & 1152 & 12 & 42 \\
$\mathrm{ZBLALiP}$ & 90 & 4.2 & 981 & 10 & 43 \\
$\mathrm{SiO}_{2}$ & Glass & 7.3 & 1289 & 13 & 40 \\
$\mathrm{SiO}_{2}$ & Glass & 1.3 & 335 & 3 & 41 \\
$\mathrm{SiO}_{2}$ & Glass & 3.6 & 592 & 6 & 44 \\
\hline \hline
\end{tabular}

TABLE III. Summary of sensitivities and techniques used for the microspheres under investigation.

\begin{tabular}{lcc}
\hline \hline & Technique & Sensitivity $\left(37{ }^{\circ} \mathrm{C}\right) \times 10^{-3}{ }^{\circ} \mathrm{C}^{-1}$ \\
\hline $\mathrm{Eu}^{3+}$ & Spectral shift & 30 \\
$\mathrm{~Tb}^{3+}$ & Band-shape & 0.7 \\
$\mathrm{Er}^{3+}$ & Band-shape (FIR) & 16 \\
\hline \hline
\end{tabular}

est value of sensitivity, due to the superior sensitivity of the $\mathrm{Eu}^{3+}:{ }^{5} \mathrm{D}_{0} \rightarrow{ }^{7} \mathrm{~F}_{2}$ transition to environmental effects. ${ }^{32,33}$ The different techniques used to obtain the ion sensitivity have the advantage that are unaffected by local intensity changes (concentration of $\mathrm{RE}^{3+}$ or laser fluctuations) and so require simple experimental instrumentation and so require simple experimental instrumentation when compared to previously proposed approaches for luminescence thermal sensing at the sub-micrometric scale. ${ }^{4}$ These results together with the possibility of trapping and handling the sub-micrometric spheres without perturbation of the medium make these spheres highly suitable as a thermal sensor in microfluidic.

\section{CONCLUSIONS}

In this work, we have evaluated the potential use of $\mathrm{RE}^{3+}$ doped $\mathrm{SiO}_{2}$ sub-micrometric spheres as optically controlled thermal sensors. We report on the synthesis of high quality rare earth $\left(\mathrm{Er}^{3+}, \mathrm{Eu}^{3+}\right.$, and $\mathrm{Tb}^{3+}$ ions) doped spheres with reasonable size dispersion and with an outstanding solubility and colloidal character in aqueous solutions. The obtained sub-micrometric spheres have shown narrow size distribution characterized by a mean diameter of $593 \mathrm{~nm}$ and a size poly-dispersity of $\sigma \approx 2 \%$. These facts allow for optical manipulation of single spheres by using laser tweezers with laser powers as low as $10 \mathrm{~mW}$ that, in turns, cause negligible local heating effects. Finally, the temperature sensitivity of the luminescence properties of the sub-micrometric spheres was systematically investigated. All the spectral properties of the $\mathrm{RE}^{3+}$ doped materials have been found to be affected by temperature variations within the biological range. Our sensitivities were compared with other $\mathrm{RE}^{3+}$ doped materials at the physiological temperature of $37^{\circ} \mathrm{C}$. For the particular case of $\mathrm{Eu}^{3+}$ doped microspheres, we have found a thermal sensitivity of $0.03{ }^{\circ} \mathrm{C}^{-1}$, which is one of the highest value reported up to now and indicates that in a near future these sub-micrometric spheres could be used as high resolution thermal sensors. For the $\mathrm{Er}^{3+}$ doped sub-micrometric spheres, the sensitivity reaches a value of $0.016{ }^{\circ} \mathrm{C}^{-1}$, which is again one of the highest reported by comparing with other microspheres and materials doped with the same rare earth ion.

According to the results presented in this work, $\mathrm{Eu}^{3+}$ and $\mathrm{Er}^{3+}$ doped silica microspheres emerge as promising optically controllable thermal sensors with potential application in thermal imaging of microfluids and living cells.

\section{ACKNOWLEDGMENTS}

This work was supported by the Universidad Autónoma de Madrid and Comunidad Autónoma de Madrid (Projects CCG087-UAM/MAT-4434 and S2009/ 
MAT- 1756), by the Spanish Ministerio de Educacion y Ciencia (MAT2010-16161 and MAT2010-21270-C04-02), FPI grant by Agencia Canaria de Investigación del Gobierno de Canarias, by the Caja Madrid Foundation and by Fondazione Cariverona, project Verona Nanomedicine Initiative.

${ }^{1}$ M. Suzuki, V. Tseeb, K. Oyama, and S. Ishiwata, Biophys. J. 92, L46 (2007).

${ }^{2}$ O. Zohar, M. Ikeda, H. Shinagawa, H. Inoue, H. Nakamura, D. Elbaum, D. L. Alkon, and T. Yoshioka, Biophys. J. 74, 82 (1998).

${ }^{3}$ C. B. Muller, K. Weiss, A. Loman, J. Enderlein, and W. Richtering, Lab Chip 9, 1248 (2009).

${ }^{4}$ D. Jaque and F. Vetrone, Nanoscale, 4, 4301 (2012).

${ }^{5}$ E. Saidi, N. Babinet, L. Lalouat, J. Lesueur, L. Aigouy, S. Volz, J. Labeguerie-Egea, and M. Mortier, Small 7, 259 (2011).

${ }^{6}$ P. Low, B. Kim, N. Takama, and C. Bergaud, Small 4, 908 (2008).

${ }^{7}$ M. M. Wang, E. Tu, D. E. Raymond, J. M. Yang, H. C. Zhang, N. Hagen, B. Dees, E. M. Mercer, A. H. Forster, I. Kariv, P. J. Marchand, and W. F. Butler, Nature Biotechnol. 23, 83 (2005).

${ }^{8}$ R. K. P. Benninger, Y. Koc, O. Hofmann, J. Requejo-Isidro, M. A. A. Neil, P. M. W. French, and A. J. deMello, Anal. Chem. 78, 2272 (2006).

${ }^{9}$ A. Ashkin, J. M. Dziedzic, and T. Yamane, Nature 330, 769 (1987).

${ }^{10}$ S. Ebert, K. Travis, B. Lincoln, and J. Guck, Opt. Express 15, 15493 (2007).

${ }^{11}$ F. Tardieu, M. Reymond, P. Hamard, C. Granier, and B. Muller, J. Exp. Bot. 51, 1505 (2000).

${ }^{12}$ J. Lee and N. A. Kotov, Nano Today 2, 48 (2007).

${ }^{13}$ F. Vetrone, R. Naccache, A. Zamarron, A. J. de la Fuente, F. Sanz-Rodriguez, L. M. Maestro, E. M. Rodriguez, D. Jaque, J. G. Sole, and J. A. Capobianco, ACS Nano 4, 3254 (2010).

${ }^{14}$ J. M. Yang, H. Yang, and L. W. Lin, ACS Nano 5, 5067 (2011).

${ }^{15}$ L. M. Maestro, E. M. Rodriguez, F. Vetrone, R. Naccache, H. L. Ramirez, D. Jaque, J. A. Capobianco, and J. G. Sole, Opt. Express 18, 23544 (2010). ${ }^{16}$ P. S. Dorozhkin, S. V. Tovstonog, D. Golberg, J. H. Zhan, Y. Ishikawa, M. Shiozawa, H. Nakanishi, K. Nakata, and Y. Bando, Small 1, 1088 (2005).

${ }^{17}$ Y. C. Lan, H. Wang, X. Y. Chen, D. Z. Wang, G. Chen, and Z. F. Ren, Adv. Mater. 21, 4839 (2009).

${ }^{18}$ M. A. Bennet, P. R. Richardson, J. Arlt, A. McCarthy, G. S. Buller, and A. C. Jones, Lab Chip 11, 3821 (2011).

${ }^{19}$ G. W. Walker, V. C. Sundar, C. M. Rudzinski, A. W. Wun, M. G. Bawendi, and D. G. Nocera, Appl. Phys. Lett. 83, 3555 (2003).

${ }^{20}$ L. M. Maestro, C. Jacinto, U. R. Silva, F. Vetrone, J. A. Capobianco, D. Jaque, and J. G. Sole, Small 7, 1774 (2011).

${ }^{21}$ H. Mushfique, J. Leach, H. B. Yin, R. Di Leonardo, M. J. Padgett, and J. M. Cooper, Anal. Chem. 80, 4237 (2008).

${ }^{22}$ A. Terray, J. Oakey, and D. W. M. Marr, Science 296, 1841 (2002).
${ }^{23}$ J. Enger, M. Goksor, K. Ramser, P. Hagberg, and D. Hanstorp, Lab Chip 4, 196 (2004).

${ }^{24}$ G. P. McNerney, W. Hubner, B. K. Chen, and T. Huser, J. Biophotonics 3, 216 (2010).

${ }^{25}$ M. Capitanio, D. Maggi, F. Vanzi, and F. S. Pavone, J. Opt. A, Pure Appl. Opt. 9, S157 (2007).

${ }^{26}$ K. Ramser, W. Wenseleers, S. Dewilde, S. Van Doorslaer, L. Moens, and D. Hanstorp, J. Biomed. Opt. 12, 044009 (2007).

${ }^{27}$ M. A. R. C. Alencar, G. S. Maciel, C. B. de Araujo, and A. Patra, Appl. Phys. Lett. 84, 4753 (2004).

${ }^{28}$ F. Vetrone, R. Naccache, A. J. de la Fuente, F. Sanz-Rodriguez, A. Blazquez-Castro, E. M. Rodriguez, D. Jaque, J. G. Sole, and J. A. Capobianco, Nanoscale 2, 495 (2010).

${ }^{29}$ W. Stober, A. Fink, and E. Bohn, J. Colloid Interface Sci. 26, 62 (1968).

${ }^{30}$ H. B. Mao, J. R. Arias-Gonzalez, S. B. Smith, I. Tinoco, and C. Bustamante, Biophys. J. 89, 1308 (2005).

${ }^{31}$ V. K. Rai, Appl. Phys. B: Lasers Opt. 88, 297 (2007).

${ }^{32}$ V. Lavin, J. E. Munoz-Santiuste, T. Troster, U. R. Rodriguez-Mendoza, J. Gonzalez-Platas, C. Gonzalez-Silgo, I. R. Martin, F. Rivera-Lopez, and S. Gonzalez-Perez, High Press. Res. 26, 411 (2006).

${ }^{33}$ M. I. J. Stich, S. Nagl, O. S. Wolfbeis, U. Henne, and M. Schaeferling, Adv. Funct. Mater. 18, 1399 (2008).

${ }^{34}$ S. V. Yap, R. M. Ranson, W. M. Cranton, and D. Koutsogeorgis, Appl. Opt. 47, 4895 (2008).

${ }^{35}$ J. B. Yu, L. N. Sun, H. S. Peng, and M. I. J. Stich, J. Mater. Chem. 20, 6975 (2010).

${ }^{36}$ P. Haro-Gonzalez, S. F. Leon-Luis, S. Gonzalez-Perez, and I. R. Martin, Mater. Res. Bull. 46, 1051 (2011).

${ }^{37}$ P. Haro-Gonzalez, I. R. Martin, L. L. Martin, S. F. Leon-Luis, C. PerezRodriguez, V. Lavin, and M. C. Team, Opt. Mater. 33, 742 (2011).

${ }^{38}$ Z. P. Cai, L. Xiao, H. Y. Xu, and M. Mortier, J. Lumin. 129, 1994 (2009).

${ }^{39}$ M. Quintanilla, E. Cantelar, F. Cusso, M. Villegas, and A. C. Caballero, Appl. Phys. Express 4, 022601 (2011).

${ }^{40}$ S. Q. Zhou, C. R. Li, Z. F. Liu, S. F. Li, and C. L. Song, Opt. Mater. 30, 513 (2007).

${ }^{41}$ C. R. Li, B. Dong, C. G. Ming, and M. K. Lei, Sensors 7, 2652 (2007).

${ }^{42}$ J. M. Ward and S. N. Chormaic, Appl. Phys. B: Lasers Opt. 100, 847 (2010).

${ }^{43}$ Z. P. Cai and H. Y. Xu, Sens. Actuators, A 108, 187 (2003).

${ }^{44}$ C. R. Li, B. Dong, S. F. Li, and C. H. Song, Chem. Phys. Lett. 443, 426 (2007).

${ }^{45}$ H. S. Peng, M. I. J. Stich, J. B. Yu, L. N. Sun, L. H. Fischer, and O. S. Wolfbeis, Adv. Mater. 22, 716 (2010).

${ }^{46}$ V. K. Rai and A. Rai, Appl. Phys. B: Lasers Opt.86, 333 (2007).

${ }^{47}$ Y. Dwivedi and S. B. Rai, Sens. Actuators, A 163, 37 (2010).

${ }^{48}$ S. V. Yap, R. M. Ranson, W. M. Cranton, D. C. Koutsogeorgis, and G. B. Hix, J. Lumin. 129, 416 (2009). 